\title{
Study of water Cherenkov detector designs for the SWGO experiment
}

Francesca Bisconti ${ }^{a, *}$ and Andrea Chiavassa ${ }^{a}$ on behalf of the SWGO Collaboration (a complete list of authors can be found at the end of the proceedings)

\author{
${ }^{a}$ University of Turin, \\ Via Pietro Giuria 1, Turin, Italy \\ E-mail: francesca.bisconti@to.infn.it
}

The Southern Wide-field Gamma-ray Observatory (SWGO) is a next-generation ground-based gamma-ray detector under development to reach a full sky coverage together with the current HAWC and LHAASO experiments in the northern hemisphere. It will complement the observation of transient and variable multi-wavelength and multi-messenger phenomena, offering moreover the possibility to access the Galactic Centre. One of the possible SWGO configurations consists of an array of water Cherenkov tanks, with a high fill-factor inner array and a low-density outer array, covering an overall area of one order of magnitude larger than HAWC. To reach a high detection efficiency and discrimination capability between gamma-ray and hadronic air showers, various tank designs were studied. Double-layer tanks with several sizes, shapes and number of photomultiplier tubes have been considered. Single-particle simulations have been performed to study the tank response, using muons, electrons, and gamma-rays with energies typical of extensive air showers particles, entering the tanks with zenith angles from 0 to 60 degrees. The tank response was evaluated considering the particle detection efficiency, the number of photoelectrons produced by the photomultiplier tubes, and the time resolution of the measurement of the first photon. The study allowed to compare the performance of tanks with circular and square base, to understand which design optimizes the performance of the array. The method used in the study and the results will be discussed in this paper.

$37^{\text {th }}$ International Cosmic Ray Conference (ICRC 2021)

July 12th - 23rd, 2021

Online - Berlin, Germany

\footnotetext{
${ }^{*}$ Presenter
} 


\section{Introduction}

The SWGO experiment $[1,2]$ is a next-generation gamma-ray observatory to be installed in the Southern Hemisphere, in South America, to be complementary to other gamma-ray experiments in the Northern Hemisphere, as HAWC [3] and LHAASO [4], for the observation of the entire sky. It will be based on ground-level particle detection, with close to $100 \%$ duty cycle and order steradian field of view. The site has to be at high altitude, in order to be closer to the maximum of the extensive air showers induced by gamma-rays. The SWGO design has to be defined [2]. However, one configuration under study consists of an array of water Cherenkov detectors (tanks) with a high fill-factor core with area considerably larger than HAWC and significantly better sensitivity, and a low density outer array.

To study the performance of single tanks, we performed simulations of particles through tanks with different size and configuration of PMTs. We considered double-layer tanks [5] with circular (Circular-DLT) and square (Square-DLT) base and several dimensions. Single-layer tanks are under consideration, too [6].

\section{Simulations}

\subsection{Particles}

In this analysis we performed simulations of electrons, gamma-rays and muons with random direction and fixed energies selected in order to reproduce the most probable ones in extensive air showers (EASs) at 4500-5000 m a.s.l., generated by $400 \mathrm{GeV}$ protons and $200 \mathrm{GeV}$ photons. Therefore, we simulated $10 \mathrm{MeV}, 100 \mathrm{MeV}$ and $1 \mathrm{GeV}$ electrons and photons, and $1 \mathrm{GeV}$ and $10 \mathrm{GeV}$ muons. The generation points lie on an area $10 \mathrm{~cm}$ above the tank and centered with the tank. For Circular-DLT, the circular area where particles are generated has a radius $10 \mathrm{~cm}$ larger than the radius of the tank. Similarly, for Square-DLT particles are generated on a square area with half-side $10 \mathrm{~cm}$ larger than the half-side of the tank. We used azimuth angles $\phi$ in the range $0-360 \mathrm{deg}$ and zenith angles $\theta$ in the range $0-60 \mathrm{deg}$ extracted from the distribution $\cos ^{2} \theta$. For each tank design, 10000 particles entering the tanks have been analyzed.

\subsection{Specifications of the tanks}

To retrieve the response of single tanks crossed by particles we used the HAWCSim framework [7], which makes use of Geant4 to simulate the interaction of the particle with the tank itself and the water, including the production of the Cherenkov photons that can be detected by the PMTs installed inside the tank. In Fig. 1 two examples of the Geant 4 visualization of a Circular-DLT and a Square-DLT crossed by a muon are shown.

\subsubsection{Dimensions of the tanks}

The height of the upper layer was chosen allowing the Cherenkov photons to reach any PMT at the base of the upper layer. Assuming a vertical particle entering the tank from the center of the roof, the Cherenkov photons should be able to reach the lateral walls of a Circular-DLT or the corners of a Square-DLT at the base of the upper layer. For Circular-DLT the height $h$ and radius $r$ follow the relation $h=r / \tan \theta_{C}$, where $\theta_{C}=41.2 \mathrm{deg}$ is the emission angle of the Cherenkov photons with 

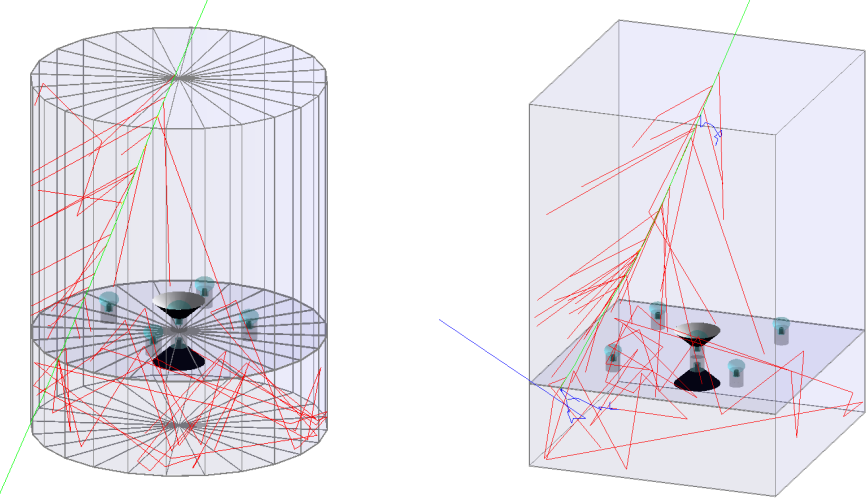

Figure 1: Simulation of a Circular-DLT and a Square-DLT crossed by a muon. The green line represents the simulated muon, the red lines optical photons, blue lines gamma-rays, and yellow lines electrons.

respect to the trajectory of the particle crossing the water. For Square-DLT, the relation between the height and the half side $l$ is $h=\sqrt{2} l / \tan \theta_{C}$. To the height calculated with previous formulas, $1 \mathrm{~m}$ of water is added to have $90 \%$ probability that gamma-rays interact by pair production. The lower layer, with height independent of the radius, is dedicated to muon measurements, increasing the gamma/hadron discrimination and allowing the separation of mass groups of charged primaries (from 2 to 4). For the lower layer, we chose heights of $0.5 \mathrm{~m}, 0.75 \mathrm{~m}$ and $1 \mathrm{~m}$. The dimensions of the tanks are collected in Tab. 1.

\begin{tabular}{lllll} 
Tank & Width $(\mathrm{m})$ & Height u.l. Cyl. $(\mathrm{m})$ & Height u.l. Sqr. $(\mathrm{m})$ & Height 1.1. $(\mathrm{m})$ \\
\hline T1 & 3 & 2.7 & 3.4 & $0.5,0.75,1$ \\
T2 & 3.5 & 3.0 & 3.8 & $0.5,0.75,1$ \\
T3 & 4 & 3.3 & 4.2 & $0.5,0.75,1$ \\
T4 & 4.5 & 3.6 & 4.6 & $0.5,0.75,1$ \\
T5 & 5 & 3.9 & 5.0 & $0.5,0.75,1$ \\
T6 & 5.5 & 4.2 & 5.4 & $0.5,0.75,1$
\end{tabular}

Table 1: Size of the tanks. "Width" is the diameter of Circular-DLT and side of Square-DLT; "Height u.l. Cyl." is the height of the upper layer of Circular-DLT; "Height u.l. Sqr." is the height of the upper layer of Square-DLT; "Height 1.1." is the height of the lower layer.

\subsubsection{Properties of the inner walls}

For the inner walls of the upper layer of the tanks, we used both reflective (Tyvek) and nonreflective (Polypropylene) materials. The reflectivity of the materials depend on the wavelength of the incident photons. Tyvek has a reflectivity of $0.63-0.92$ in the range of wavelength $250-650 \mathrm{~nm}$; polypropylene has a reflectivity of 0.10 over the same range on wavelength. Reflective walls allow for a better detection capability, but might extend the detection time, due to possible consecutive reflections of photons on the walls before they reach the PMTs. This would result in a higher detection efficiency and a lower time resolution for the detection of the first photon. For the lower 
layer we used reflective walls, as the priority was given to the detection efficiency of particles entering the lower layer rather than the timing.

\subsubsection{PMTs}

In the upper layer we used two configurations of PMTs looking upwards: one central 10" PMT or four peripheral 5" PMTs placed at half radius in the Circular-DLT and half diagonal in the Square-DLT (whose signals are summed in one unique output); in the lower layer we used one central 10" PMT and 5" PMT looking downwards. In each layer, the two PMT configurations have to be considered independently. In HAWCSim three models of PMT from Hamamatsu were available at the time of this analysis: 8" R5912 PMT, 10" R7081HQE PMT, and 3" R12199 PMT. To simulate the four 5" PMTs in the upper layer, the 8" R5912 PMTs were initially simulated, and then rescaled to 5 " PMT during the analysis phase.

\subsection{Analysis}

We evaluated the tanks response considering the number of photoelectrons (PEs) produced by the PMTs, the particle detection efficiency (at 1 and 2 PEs threshold level), and the measurement resolution of the first photon. For the analysis of the upper layer, we analyzed the distributions

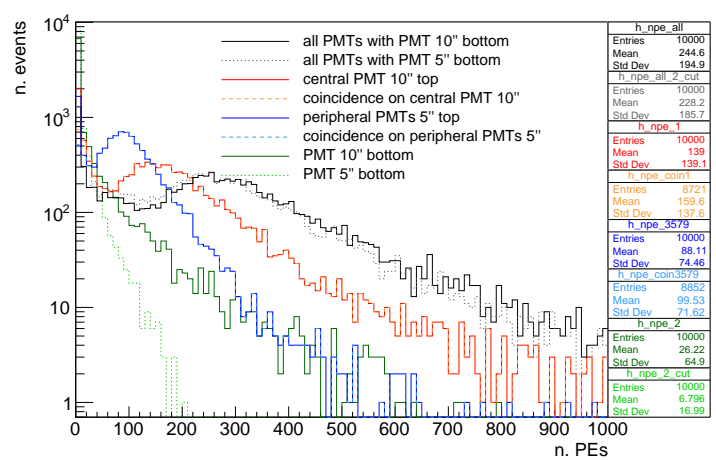

(a) gamma-rays - PEs distribution

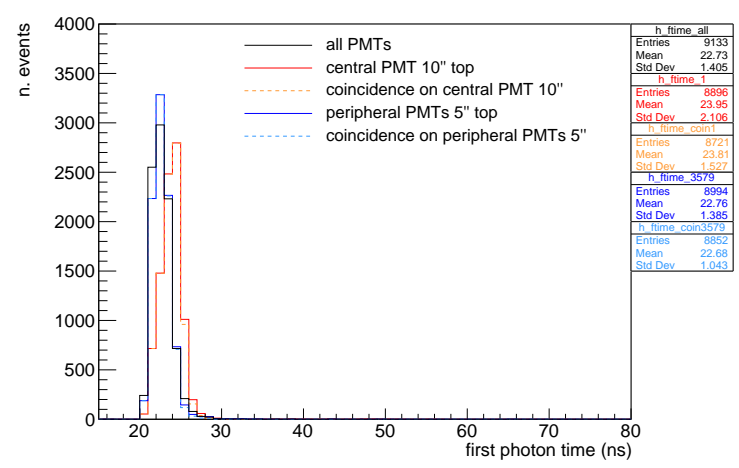

(c) gamma-rays - time first photon

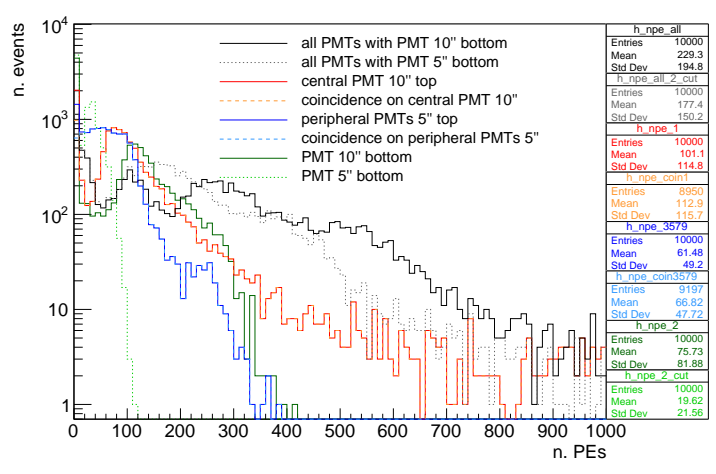

(b) muons - PEs distribution

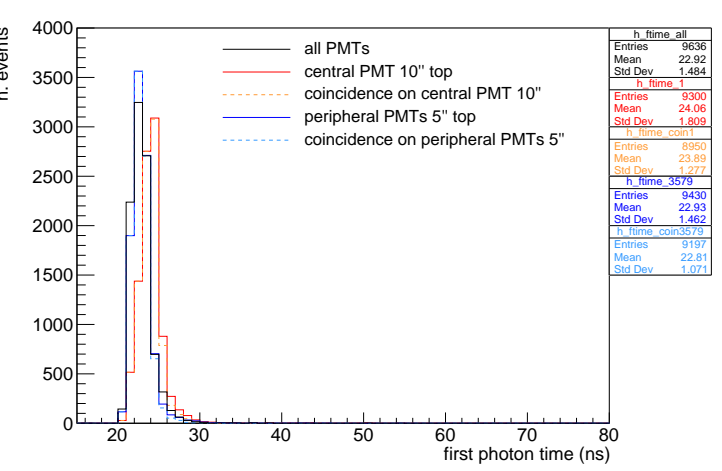

(d) muons - time first photon

Figure 2: Distributions of the number of PEs and of the arrival time of the first photon.

of the number of PEs and the distribution of the arrival time of the first photon, produced by the 
central 10" PMT and by the sum of the four peripheral 5" PMTs, separately. We also considered a threshold of 2 PEs arriving within 30 ns for the central 10" PMT and for the sum of the four peripheral 5" PMTs. The time resolution of the measurement of the first photon has been estimated from the standard deviation of the distribution of the time of the first photon. For the lower layer, we analyzed the distributions of the number of PEs produced by the 10" PMT and the 5" PMT, separately. This allowed to understand which of the two configurations in the upper layer gives the higher detection efficiency and the better time resolution of the first detected photon and, for the lower layer, how a different size of the PMT changes the detection efficiency.

In Fig. 2 sample distributions of the number of PEs (a-b) and of the arrival time of the first photon (c-d) are shown. They refer to simulations of $1 \mathrm{GeV}$ particles crossing Circular-DLT with lower layer $1 \mathrm{~m}$ high, non-reflective walls in the upper layer and reflective walls in the lower layer. The plots on the left side are for gamma-rays (the distributions for electrons are similar to those for gamma-rays) and the plots on the right side are for muons. In the distribution of the number of PEs in the lower layer, the contribution due to muons is evident, while for gamma-rays it is not. The reason is that muons can reach the lower layer with a higher probability than gamma-rays. The PE distributions also show that particles generate in average more PEs on the central 10" PMT than on the four peripheral 5" PMTs. However, the number of particles generating at least $1 \mathrm{PE}$ via the two configurations of PMTs are comparable. The same is valid also considering a threshold of 2 PEs. The distributions of the arrival time of the first photon are similar. In the example reported, the timing resolution for the four peripheral PMTs is slightly narrower than for the central PMT.

\section{Results}

The parameters taken into account to compare the tank performance are:

- The number of PEs produced in both layers. In the upper layer we considered separately the configuration with one central 10" PMT and four peripheral 5" PMTs. For the lower layer we considered a central 10" PMT or 5" PMT.

- The time resolution of the measurement of the first photon in the upper layer, evaluated as the standard deviation of the distribution of the first photon arrival time.

- The detection efficiency of both layers. For the upper layer, the efficiency is calculated as the number of particles (events) that produce at least 1 PE or 2 PEs (depending on the threshold), in the central 10" PMT or in the four peripheral 5" PMTs, divided by the total number of particles entering the tank (10000). For the lower layer, we used the same method but considering only $1 \mathrm{PE}$ threshold.

In the following plots, electrons are not displayed as they give similar results to those for gamma-rays.

In both Circular-DLT and Square-DLT designs, the number of detected PEs and consequently the detection efficiency decrease while increasing the size of the tank (see Fig. 3). This is due to the decrease of the ratio between the area of the PMT and that of the base of the tank. We made some test simulations using different tank widths and rescaling the PMT size in order to have a constant ratio between the area of the PMT and the area of the tank's base, and the detection efficiency resulted to be almost constant. 


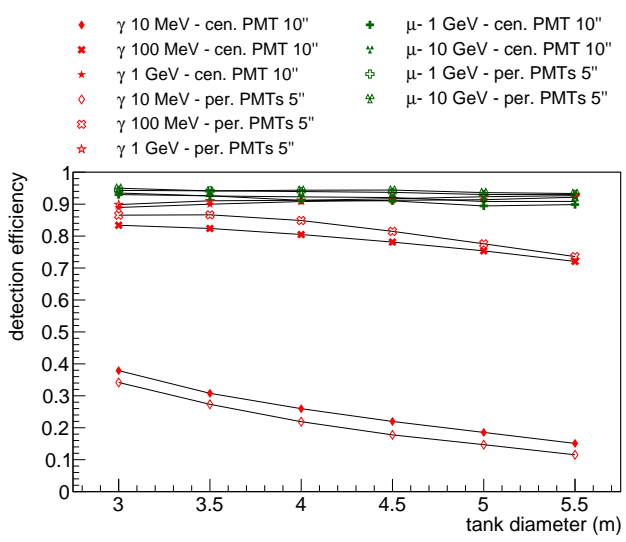

(a) Circular-DLT 1 PE threshold

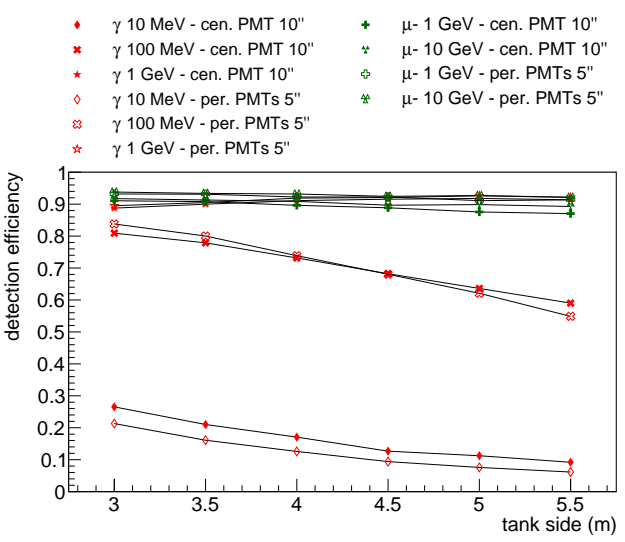

(b) Square-DLT 1 PE threshold

Figure 3: Detection efficiency of the upper layer with non-reflective walls.

For the upper layer, the performance of Circular-DLT is slightly better than that of Square-DLT, in terms of number of PEs and detection efficiency (see Fig. 3 (a) and (b)). Considering upper layers with reflective walls, the detection efficiency becomes higher, especially for electrons and gamma-rays with energy $10 \mathrm{MeV}$ (see Fig. 3 and Fig. 4).

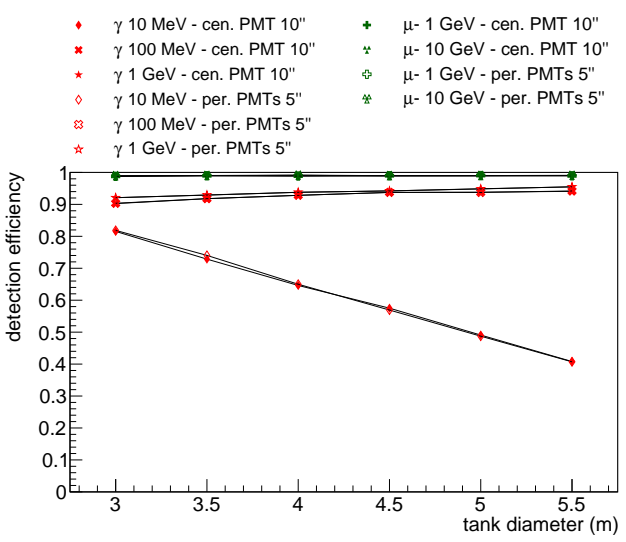

(a) Circular-DLT 1 PE threshold

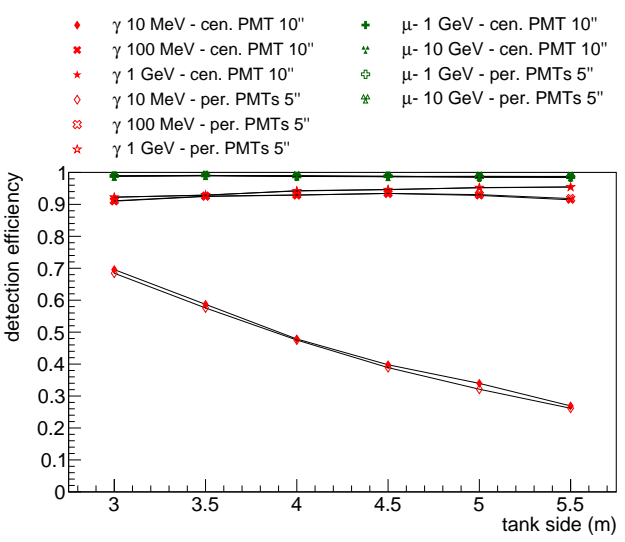

(b) Square-DLT 1 PE threshold

Figure 4: Detection efficiency of the upper layer with reflective walls.

The time resolution of the measurement of the first photon is similar for Circular-DLT and Square-DLT (see Fig. 5 (a) and (b)), but using reflective walls it becomes about two times larger for $100 \mathrm{MeV}$ and $1 \mathrm{GeV}$ particles, and strongly increases for particles of $10 \mathrm{MeV}$, because the time distribution of the first photon shows a long tail for these particles (see Fig. 5 and Fig. 6). The detection efficiency of the upper layers considering one central 10" PMT or four peripheral 5" PMTs are comparable in small tanks, although more PEs are produced in the central PMT.

For the lower layer, the number of PEs and the detection efficiency are slightly higher in Circular-DLT than in Square-DLT (see Fig. 7 (a) and (b)). Electrons and gamma-rays of $10 \mathrm{MeV}$ and $100 \mathrm{MeV}$ are rarely detected in lower layers. The height of the lower layer influences the number of PEs, which is lower for $0.5 \mathrm{~m}$ and comparable for $0.75 \mathrm{~m}$ and $1 \mathrm{~m}$, but does not affect the 


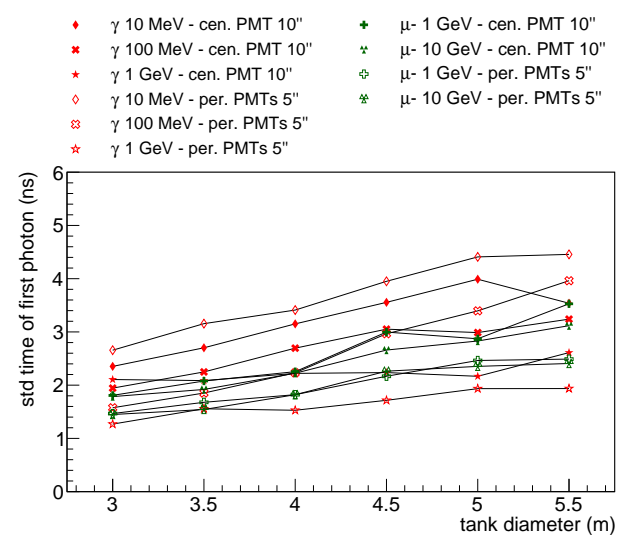

(a) Circular-DLT 1 PE threshold

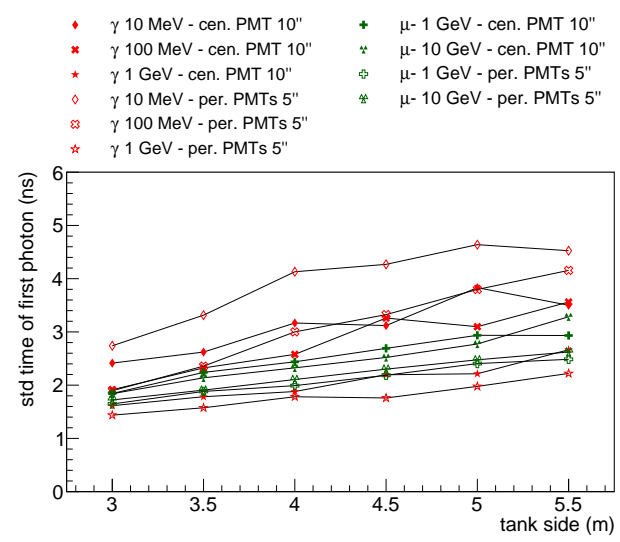

(b) Square-DLT 1 PE threshold

Figure 5: Time resolution of the measurement of the first photon in the upper layer with non-reflective walls.

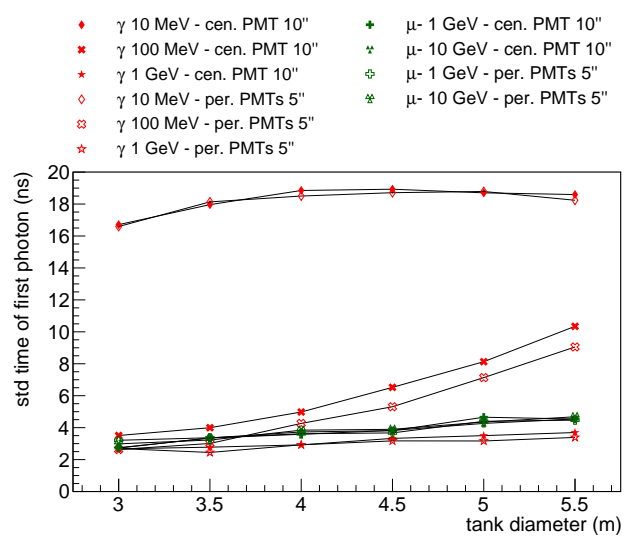

(a) Circular-DLT 1 PE threshold

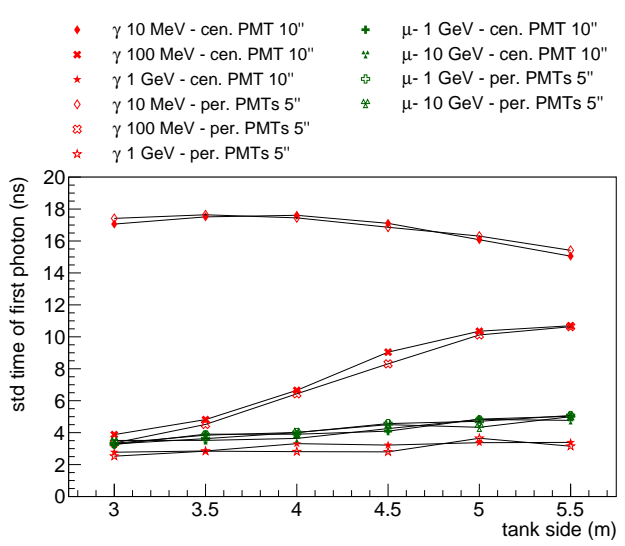

(b) Square-DLT 1 PE threshold

Figure 6: Time resolution of the measurement of the first photon in the upper layer with reflective walls.

detection efficiency. Considering a 5" PMT instead of a 10" PMT, the efficiency slightly decreases although the number of produced PEs is the $25 \%$, proportional to the area of the photocathode.

\section{Conclusion}

This study allowed to compare tanks with circular and square base of different size, and tanks with reflective and non-reflective walls in the upper layer.

We found that regardless of the tank design and reflective properties of the upper layer, the performance of the tanks worsen while increasing the width of the tank, because the "sensitive area", i.e. the area covered by the PMTs, decreases with respect to that of the base of the tank.

Circular-DLT have slightly better performance with respect to Square-DLT. Nevertheless, for the final design of the SWGO array we should take into account also that with Square-DLT it is possible to achieve a higher fill factor, although they are a potentially more expensive solution.

By using reflective walls instead of non-reflective walls in the upper layers, the detection efficiency increases, but the time resolution of the measurement of the first photon widen, in 


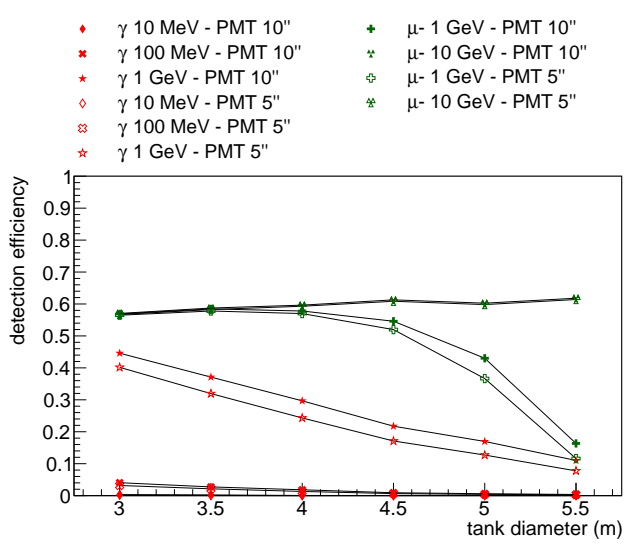

(a) Circular-DLT

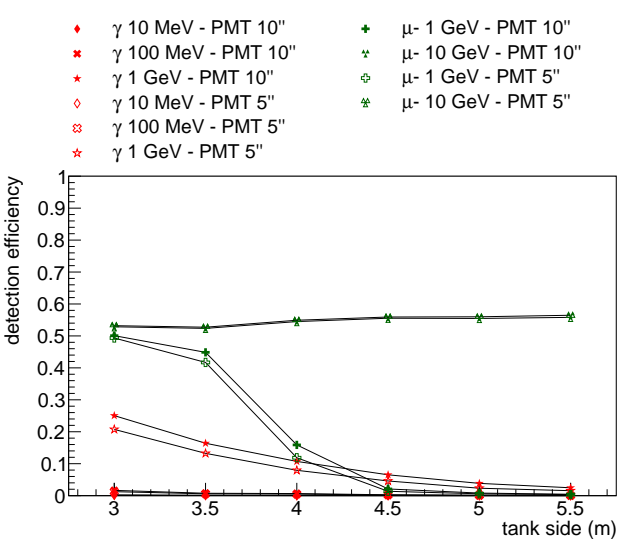

(b) Square-DLT

Figure 7: Detection efficiency of the lower layer.

particular for particle with low energy.

We plan to run similar simulations with double-layer tanks with hexagonal base, to complete the study of the single tanks performance with different tank designs.

\section{Acknowledgements}

The SWGO Collaboration acknowledges the support from the agencies and organizations listed here: https://wWW.swgo.org/SWGOWiki/doku.php?id=acknowledgements.

\section{References}

[1] U. Barres de Almeida (for the SWGO Collaboration), "The Southern Wide-Field Gamma-ray Observatory (SWGO)”, arXiv e-prints: arXiv:2012.13740 (2020)

[2] J. Hinton et al. (for the SWGO Collaboration), "The Southern Wide-field Gamma-ray Observatory: Status and Prospects" - this conference (2021)

[3] T. DeYoung (for the HAWC Collaboration), "The HAWC observatory", Nuclear Instruments and Methods in Physics Research A, 692, 72 (2012)

[4] X. Bai et al., "The Large High Altitude Air Shower Observatory (LHAASO) Science White Paper", arXiv e-prints: arXiv:1905.02773 (2019)

[5] S. Kunwar et al. (for the SWGO Collaboration), "Double-layered Water Cherenkov Detector for the Southern Wide-field-of-view Gamma-ray Observatory (SWGO)" - this conference (2021)

[6] R. Conceição et al. (for the SWGO Collaboration), "Gamma/hadron discrimination using a small-WCD with four PMTs" - this conference (2021)

[7] H. Schoorlemmer et al. (for the SWGO Collaboration), "Simulating the performance of the Southern Wide-view Gamma-ray Observatory" - this conference (2021) 


\section{Full Authors List: SWGO Collaboration}

P. Abreu ${ }^{1}$, A. Albert ${ }^{2}$, E. O. Angüner ${ }^{3}$, C. Arcaro $^{4}$, L.H. Arnaldi ${ }^{5}$, J.C. Arteaga-Velázquez ${ }^{6}$, P. Assis ${ }^{1}$, A. Bakalová ${ }^{7}$, U. Barres de Almeida ${ }^{8}$, I. Batković ${ }^{4}$, J. Bellido ${ }^{9}$, E. Belmont-Moreno ${ }^{10}$, F. Bisconti ${ }^{11}$, A. Blanco ${ }^{1}$, M. Bohacova ${ }^{7}$, E. Bottacini ${ }^{4}$, T. Bretz ${ }^{12}$, C. Brisbois ${ }^{13}$, P. Brogueira ${ }^{1}$, A.M. Brown ${ }^{14}$, T. Bulik ${ }^{15}$, K. S. Caballero Mora ${ }^{16}$, S. M. Campos ${ }^{17}$, A. Chiavassa ${ }^{11}$, L. Chytka ${ }^{7}$, R. Conceição ${ }^{1}$, G. Consolati ${ }^{18}$, J. Cotzomi Paleta ${ }^{19}$, S. Dasso ${ }^{20}$, A. De Angelis ${ }^{4}$, C.R. De Bom ${ }^{8}$, E. de la Fuente ${ }^{21}$, V. de Souza ${ }^{22}$, D. Depaoli ${ }^{11}$, G. Di Sciascio ${ }^{23}$, C. O. Dib ${ }^{24}$, D. Dorner ${ }^{25}$, M. Doro ${ }^{4}$, M. Du Vernois ${ }^{26}$, T. Ergin $^{27}$, K. L. Fan ${ }^{13}$, N. Fraija ${ }^{8}$, S. Funk ${ }^{28}$, J. I. García ${ }^{17}$, J. A. García-González ${ }^{29}$, S. T. García Roca ${ }^{9}$, G. Giacinti ${ }^{30}$, H. Goksu ${ }^{30}$, B. S. González ${ }^{1}$, F. Guarino ${ }^{31}$, A. Guillén ${ }^{32}$,

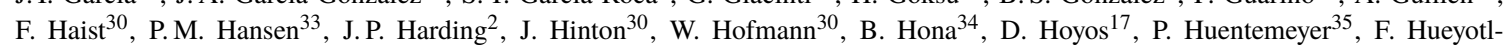
Zahuantitla $^{16}$ A. Insolia ${ }^{36}$, P. Janecek ${ }^{7}$, V. Joshi ${ }^{28}$, B. Khelifi ${ }^{37}$, S. Kunwar ${ }^{30}$, G. La Mura ${ }^{1}$, J. Lapington ${ }^{38}$, M. R. Laspiur ${ }^{17}$, F. Leitl ${ }^{28}$, F. Longo ${ }^{39}$, L. Lopes ${ }^{1}$, R. Lopez-Coto ${ }^{4}$, D. Mandat ${ }^{7}$, A. G. Mariazzi ${ }^{33}$, M. Mariotti ${ }^{4}$, A. Marques Moraes ${ }^{8}$, J. Martínez-Castro ${ }^{40}$, H. Martínez-Huerta ${ }^{41}$, S. May ${ }^{42}$, D. G. Melo ${ }^{43}$, L. M. Mendes ${ }^{1}$, T. Mineeva ${ }^{24}$, A. Mitchell14, S. Mohan ${ }^{35}$, O. G. Morales Olivares ${ }^{16}$, E. Moreno-Barbosa ${ }^{19}$, L. Nellen ${ }^{45}$, V. Novotny ${ }^{7}$, L. Olivera-Nieto ${ }^{30}$, E. Orlando ${ }^{39}$, M. Pech $^{7}$, A. Pichel ${ }^{20}$, M. Pimenta ${ }^{1}$, M. Portes de Albuquerque ${ }^{8}$, E. Prandini ${ }^{4}$, M. S. Rado Cuchills ${ }^{9}$, A. Reisenegger ${ }^{46}$, B. Reville ${ }^{30}$, C. D. Rho ${ }^{47}$, A. C. Rovero ${ }^{20}$, E. Ruiz-Velasco ${ }^{30}$, G. A. Salazar ${ }^{17}$, A. Sandoval ${ }^{10}$, M. Santander ${ }^{42}$, H. Schoorlemmer ${ }^{30}$, F. Schüssler ${ }^{48}$, V.H. Serrano ${ }^{17}$, R. C. Shellard ${ }^{8}$, A. Sinha ${ }^{49}$, A. J. Smith ${ }^{13}$, P. Surajbali ${ }^{30}$, B. Tomé 1 , I. Torres Aguilar ${ }^{50}$, C. van Eldik $^{28}$, I. D. Vergara-Quispe ${ }^{33}$, A. Viana ${ }^{22}$, J. Vícha ${ }^{7}$, C. F. Vigorito ${ }^{11}$, X. Wang ${ }^{35}$, F. Werner ${ }^{30}$, R. White ${ }^{30}$, M. A. Zamalloa Jara ${ }^{9}$

\footnotetext{
${ }^{1}$ Laboratório de Instrumentação e Física Experimental de Partículas (LIP), Av. Prof. Gama Pinto 2, 1649-003 Lisboa, Portugal

${ }^{2}$ Physics Division, Los Alamos National Laboratory, P.O. Box 1663, Los Alamos, NM 87545, United States

${ }^{3}$ Aix Marseille Univ, CNRS/IN2P3, CPPM, 163 avenue de Luminy - Case 902, 13288 Marseille cedex 09, France

${ }^{4}$ University of Padova, Department of Physics and Astronomy \& INFN Padova, Via Marzolo 8 - 35131 Padova, Italy

${ }^{5}$ Centro Atómico Bariloche, Comisión Nacional de Energía Atómica, S. C. de Bariloche (8400), RN, Argentina

${ }^{6}$ Universidad Michoacana de San Nicolás de Hidalgo, Calle de Santiago Tapia 403, Centro, 58000 Morelia, Mich., México

${ }^{7}$ FZU, Institute of Physics of the Czech Academy of Sciences, Na Slovance 1999/2, 18200 Praha 8, Czech Republic

${ }^{8}$ Centro Brasileiro de Pesquisas Físicas, R. Dr. Xavier Sigaud, 150 - Rio de Janeiro - RJ, 22290-180, Brazil

${ }^{9}$ Academic Department of Physics - Faculty of Sciences - Universidad Nacional de San Antonio Abad del Cusco (UNSAAC), Av. de la Cultura, 733, Pabellón C-358, Cusco, Peru

${ }^{10}$ Instituto de Física, Universidad Nacional Autónoma de México, Sendero Bicipuma, C.U., Coyoacán, 04510 Ciudad de México, CDMX, México

${ }^{11}$ Dipartimento di Fisica, Università degli Studi di Torino, Via Pietro Giuria 1, 10125, Torino, Italy

12 RWTH Aachen University, Physics Institute 3, Otto-Blumenthal-Straße, 52074 Aachen, Germany

${ }^{13}$ University of Maryland, College Park, MD 20742, United States

${ }^{14}$ Durham University, Stockton Road, Durham, DH1 3LE, United Kingdom

${ }_{15}$ Astronomical Observatory, University of Warsaw, Aleje Ujazdowskie 4, 00478 Warsaw, Poland

${ }^{16}$ Facultad de Ciencias en Física y Matemáticas UNACH, Boulevard Belisario Domínguez, Km. 1081, Sin Número, Terán, Tuxtla Gutiérrez, Chiapas, México

${ }^{17}$ Facultad de Ciencias Exactas, Universidad Nacional de Salta, Avda. Bolivia No 5150, (4400) Salta Capital, Argentina

${ }^{18}$ Department of Aerospace Science and Technology, Politecnico di Milano, Via Privata Giuseppe La Masa, 34, 20156 Milano MI, Italy

${ }^{19}$ Facultad de Ciencias Físico Matemáticas, Benemérita Universidad Autónoma de Puebla, C.P. 72592, México

${ }^{20}$ Instituto de Astronomia y Fisica del Espacio (IAFE, CONICET-UBA), Casilla de Correo 67 - Suc. 28 (C1428ZAA), Ciudad

Autónoma de Buenos Aires, Argentina

${ }^{21}$ Universidad de Guadalajara, Blvd. Gral. Marcelino García Barragán 1421, Olímpica, 44430 Guadalajara, Jal., México

22 Instituto de Física de São Carlos, Universidade de São Paulo, Avenida Trabalhador São-carlense, no 400, Parque Arnold Schimidt CEP 13566-590, São Carlos - São Paulo - Brasil

${ }^{23}$ INFN - Roma Tor Vergata and INAF-IAPS, Via del Fosso del Cavaliere, 100, 00133 Roma RM, Italy

${ }^{24}$ Dept. of Physics and CCTVal, Universidad Tecnica Federico Santa Maria, Avenida España 1680, Valparaíso, Chile

${ }^{25}$ Universität Würzburg, Institut für Theoretische Physik und Astrophysik, Emil-Fischer-Str. 31, 97074 Würzburg, Germany

${ }^{26}$ Department of Physics, and the Wisconsin IceCube Particle Astrophysics Center (WIPAC), University of Wisconsin, 222 West Washington Ave., Suite 500, Madison, WI 53703, United States

27 TUBITAK Space Technologies Research Institute, ODTU Campus, 06800, Ankara, Turkey

${ }^{28}$ Friedrich-Alexander-Universität Erlangen-Nürnberg, Erlangen Centre for Astroparticle Physics, Erwin-Rommel-Str. 1, D 91058 Erlangen, Germany

${ }^{29}$ Tecnologico de Monterrey, Escuela de Ingeniería y Ciencias, Ave. Eugenio Garza Sada 2501, Monterrey, N.L., 64849, México

${ }^{30}$ Max-Planck-Institut für Kernphysik, P.O. Box 103980, D 69029 Heidelberg, Germany

${ }^{31}$ Università di Napoli "Federico II", Dipartimento di Fisica "Ettore Pancini”, and INFN Napoli, Complesso Universitario di Monte Sant'Angelo - Via Cinthia, 21 - 80126 - Napoli, Italy

${ }^{32}$ University of Granada, Campus Universitario de Cartuja, Calle Prof. Vicente Callao, 3, 18011 Granada, Spain
} 
${ }^{33}$ IFLP, Universidad Nacional de La Plata and CONICET, Diagonal 113, Casco Urbano, B1900 La Plata, Provincia de Buenos Aires, Argentina

34 University of Utah, 201 Presidents' Cir, Salt Lake City, UT 84112, United States

${ }^{35}$ Michigan Technological University, 1400 Townsend Drive, Houghton, MI 49931, United States

${ }^{36}$ Dipartimento di Fisica e Astronomia "E. Majorana", Catania University and INFN, Catania, Italy

${ }^{37}$ APC-IN2P3/CNRS, Université de Paris, Bâtiment Condorcet, 10 rue A.Domon et Léonie Duquet, 75205 PARIS CEDEX 13, France

${ }^{38}$ University of Leicester, University Road, Leicester LE1 7RH, United Kingdom

${ }^{39}$ Department of Physics, University of Trieste and INFN Trieste, via Valerio 2, I-34127, Trieste, Italy

${ }^{40}$ Centro de Investigación en Computación, Instituto Politécnico Nacional, Av. Juan de Dios Bátiz S/N, Nueva Industrial Vallejo, Gustavo A. Madero, 07738 Ciudad de México, CDMX, México

${ }^{41}$ Department of Physics and Mathematics, Universidad de Monterrey, Av. Morones Prieto 4500, San Pedro Garza García 66238, N.L., México

42 Department of Physics and Astronomy, University of Alabama, Gallalee Hall, Tuscaloosa, AL 35401, United States

${ }^{43}$ Instituto de Tecnologías en Detección y Astropartículas (CNEA-CONICET-UNSAM), Av. Gral Paz 1499 - San Martín - Pcia. de Buenos Aires, Argentina

${ }^{44}$ Department of Physics, ETH Zurich, CH-8093 Zurich, Switzerland

45 Instituto de Ciencias Nucleares, Universidad Nacional Autónoma de México (ICN-UNAM), Cto. Exterior S/N, C.U., Coyoacán, 04510 Ciudad de México, CDMX, México

${ }^{46}$ Departamento de Física, Facultad de Ciencias Básicas, Universidad Metropolitana de Ciencias de la Educación, Av. José Pedro Alessandri 774, Ñuñoa, Santiago, Chile

${ }^{47}$ Department of Physics, University of Seoul, 163 Seoulsiripdaero, Dongdaemun-gu, Seoul 02504, Republic of Korea

${ }^{48}$ Institut de recherche sur les lois fondamentales de l'Univers (IRFU), CEA, Université Paris-Saclay, F-91191 Gif-sur-Yvette, France

${ }^{49}$ Laboratoire Univers et Particules de Montpellier, CNRS, Université de Montpelleir, F-34090 Montpellier, France

${ }^{50}$ Instituto Nacional de Astrofísica, Óptica y Electrónica (INAOE), Luis Enrique Erro 1, Puebla, México 